\title{
Études de quelques plantes thérapeutiques utilisées dans le traitement de l'hypertension artérielle et du diabète : deux maladies émergentes en Côte d'Ivoire
}

\author{
Fézan H. Tra Bi", Guy M. Irié, Kohué C.C. N'Gaman \& Clejesson H.B. Mohou \\ UFR des Sciences de la Nature, Université d'Abobo-Adjamé, 02 BP 801 Abidjan 02 \\ 'Auteurpourlescorrespondances (E-mail:trabi_fezan@hotmail.com) \\ Reçu le 21 - 02 - 2008, accepté le 12-05-2008.
}

\begin{abstract}
Résumé
Des enquêtes ethnobotaniques réalisées sur les marchés d'Abidjan (Côte d'lvoire) ont permis d'inventorier 58 espèces de plantes commercialisées pour soigner 19 maladies courantes. Trente neuf (39) de ces plantes sont utilisées contre l'hypertension artérielle et le diabète. Sur les 39 plantes, 27 sont antihypertensives, 19 sont antidiabétiques et 6 plantes sont utilisées pour traiter aussi bien l'hypertension artérielle que le diabète. Il s'agit de Catharanthus roseus (L.) G. Don, Ageratum conyzoides L., Vernonia colorata (Willd.) Drake, Alchornea cordifolia (Schum. \& Thonn.) Müll. Arg., Phyllanthus amarus Schum. \& Thonn. et Parkia biglobosa (Jacq.) Benth.
\end{abstract}

Mots clefs : Enquêtes ethnomédicinales, plantes médicinales, hypertension artérielle, diabète, Abidjan, Côte d'Ivoire.

\begin{abstract}
Study of some therapeutic plants used to treat arterial hypertension and diabetes: two emergent diseases in Côte d'lvoire

Investigations on medicinal plants in the markets of Abidjan (Côte d'lvoire) allowed the inventory of 58 species of plants used to treat 19 current diseases. Thirty-nine (39) of these plants are used against arterial hypertension and diabetes. Among the 39 plants, 27 are antihypertensive, 19 are antidiabetic and 6 plants are used to treat arterial hypertension as well as diabetes. These plants are Catharanthus roseus (L.) G Don, Ageratum conyzoides $L$, Vernonia colorata (Willd.) Drake, Alchornea cordifolia (Schum. \& Thonn.) Müll. Arg., Phyllanthus amarus Schum. \& Thonn. and Parkia biglobosa (Jacq.) Benth.
\end{abstract}

Key words: Ethnomedicinal survey, medicinal plants, arterial hypertension, diabetes, Abidjan, Côte d'Ivoire.

\section{Introduction}

Les populations africaines et particulièrement celles vivant en Côte d'Ivoire, sont confrontées à l'émergence de maladies chroniques dont le traitement et le suivi constituent, pour elles, un problème économique supplémentaire. Le diabète, l'hypertension artérielle et le cancer font partie des maladies dites nouvelles, pour la médecine traditionnelle africaine. Autrefois inconnues, ces maladies ont de plus en plus pris de l'ampleur jusqu'à devenir un véritable problème de santé publique. Aujourd'hui encore, il est difficile d'obtenir des données précises sur la prévalence de ces maladies dans la plupart des pays en développement.

En Côte d'Ivoire, Comoé et al. (1993) situaient la prévalence de l'hypertension artérielle à 13,4\% dans la population abidjanaise. Depuis environ une dizaine d'années, 40 à $50 \%$ des patients de I'Institut de Cardiologie d'Abidjan présentent cette affection et $14 \%$ de la population ivoirienne en 
souffrent (Anonyme, 2003). La prévalence de l'hypertension artérielle en Côte d'Ivoire est généralement estimée à 8 - $12 \%$ et concernait, en $2000,13 \%$ de la population avec $21 \%$ pour la seule ville d'Abidjan (Koffi, 2007).

En moins d'un quart de siècle, le diabète, surtout la forme sucrée, présente sous diverses formes, est devenu un problème de santé publique dans les pays en développement. En Côte d'Ivoire, les données de 1979 indiquaient déjà une prévalence nationale de 5,7\% (Zmour, 1979 cité par Oga et al., 2006). Les travaux de Oga et al. (2006) indiquent un net accroissement des patients diabétiques entre 1979 et 2000.

Face à l'expansion de ces maladies dont la prise en charge est élevée, l'OMS, dans sa résolution AFR/RC50/R3 du 31 août 2000, encourageait les pays africains à élaborer des stratégies régionales sur la médecine traditionnelle afin d'entreprendre des recherches sur les plantes médicinales et de promouvoir leurs utilisations optimales dans les systèmes de prestation des soins de santé.

La présente étude, faite dans ce sens, est un travail préliminaire que nous avons entrepris, basé sur des enquêtes ethnomédicinales en vue de recenser sur les marchés de la ville d'Abidjan (Côte d'Ivoire), les plantes utilisées dans le traitement de l'hypertension artérielle et du diabète.

\section{Matériel et méthodes}

Les investigations ont été menées par des approches ethnobotaniques auprès des acteurs de la médecine traditionnelle, surtout des commerçantes de plantes médicinales sur les marchés du District d'Abidjan. Mais avant, nous avons cherché à nommer les pathologies dans les langues des différentes personnes avec qui nous avons travaillé puis nous avons déterminé la conception que ces acteurs ont du diabète et de l'hypertension artérielle. L'étude a été réalisée dans trois communes de la ville d'Abidjan que sont Yopougon, Abobo et Port Bouët, sélectionnées parce qu'elles constituent les principales entrées Ouest, Nord et Est de ravitaillement des marchés de la ville, en plantes médicinales (Figure 1). Dans chacune des communes, nous avons travaillé dans trois marchés choisis au hasard et dans chacun d'eux, nous avons travaillé avec trois vendeuses de plantes médicinales également choisies au hasard.

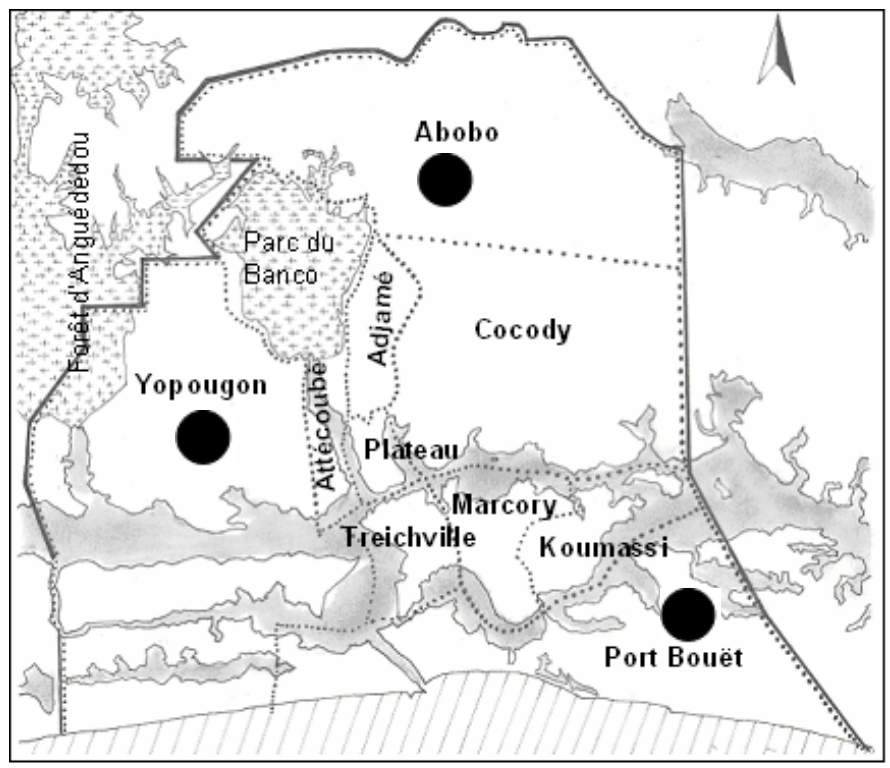

Lagune Ebrié $\mathbb{Z}$ Océan atlantique $\quad 4$ Forèt $\quad 4$

Communes visitées

Figure 1: Carte de la ville d'Abidjan indiqant les communes (cercles noirs) où les enquêtes ethnomédicines ont eu lieu (Zoro Bi \& Kouakou, 2004) 
Pendant 29 jours étalés sur 3 mois (décembre 2005 à février 2006), nous avons, dans un premier temps, recensé toutes les plantes observées sur l'étalage de chaque commerçante. Cela nous a donné une idée globale des plantes commercialisées pendant cette période de l'année dans les trois communes cibles. Des enquêtes ethnomédicinales ont, par la suite, permis de recenser les plantes médicinales commercialisées et les pathologies qui leur sont associées. Ces enquêtes consistaient à suivre les clients chez chaque vendeuse. Pour chaque client, nous avons noté la ou les plante(s) achetée(s), la ou les pathologie(s) pour laquelle elle(s) est (sont) recherchée(s), le mode de préparation de la plante, la prescription pour soigner la maladie et la durée de traitement. Ces informations nous ont permis de faire ressortir les plantes les plus recherchées et les pathologies dominantes pendant la période notre étude. Pour l'hypertension artérielle et le diabète, nous avons évalué les proportions des plantes commercialisées par le rapport des plantes effectivement achetées pour traiter la maladie sur le nombre total de plantes inventoriées au cours de l'étude, le tout ramené à 100 . Les plantes obtenues ont servi à confectionner un herbier de plantes médicinales dont la détermination a été confirmée par le Professeur Aké-Assi Laurent (Centre National de Floristique de l'Université de Cocody-Abidjan). Les différentes espèces ont été nommées selon la nomenclature de Hutchinson et Dalziel $(1954 ; 1972)$ revue par Lebrun et Stork (1991; 1992 ; 1995 ; 1997).

\section{Résultats et Discussion}

Pour réaliser cette étude, nous avons travaillé avec 27 femmes vendeuses de plantes médicinales et 493 personnes qui recherchaient au moins une plante pour se soigner. L'âge des commerçantes varie de 30 à 55 ans alors que celui des clients est compris entre 22 et 57 ans. Nous avons recensé quatre vingt trois (83) personnes qui sollicitaient des plantes contre l'hypertension artérielle et le diabète. Sur ces 83 personnes, 57 recherchaient les plantes contre I'hypertension tandis que 26 sollicitaient des plantes contre le diabète. Ce qui représente, respectivement, $11,56 \%$ d'hypertendus et 5,27 $\%$ de diabétiques sur l'ensemble de personnes sollicitant une plante pour se soigner. Ces chiffres traduisent quelque peu la prévalence de ces deux maladies dans la ville d'Abidjan, I'hypertension artérielle étant plus diagnostiquée eu égard aux attaques cardiovasculaires dont sont victimes les patients, alors que le diabète, pour son caractère silencieux, ne fait pas toujours l'objet de consultations préventives.

Notre étude a permis d'inventorier 58 espèces de plantes commercialisées dans les 3 communes de la ville d'Abidjan (Tableau 1). Elles se répartissent entre 56 genres et 33 familles. Les familles les plus représentées sont les Euphorbiaceae ( 6 espèces), les Asteraceae (5 espèces) et les Poaceae (4 espèces), viennent ensuite les Anacardiaceae, les Caesalpiniaceae, les Apocynaceae et les Rubiaceae (3 espèces). Cette représentativité a également été observée, à quelques différences près, au cours des enquêtes ethnomédicinales réalisées dans d'autres régions du pays par Kamanzi Atindehou et al. (2002) et par Diehl (2004). Cela s'explique par le fait que ces familles font partie, en nombre de genres et d'espèces, des plus importantes de la flore ivoirienne (Aké Assi, 1984; Kouamé, 1998). Ailleurs en Afrique, cette prédominance a été observée pour les familles des Euphorbiaceae et Asteraceae en Ouganda par Hamill (2003), Kamatenesi-Mugisha et Oryem-Origa (2007) puis pour les Asteraceae par Fennell et al. (2004) en Afrique du Sud. Ces trois familles de plantes figurent bien parmi celles qui procurent le plus de plantes à la pharmacopée africaine.

Toutes ces plantes sont recherchées pour traiter 19 pathologies parmi lesquelles le paludisme est la maladie qui prévaut. Ces résultats traduisent quelque peu le profil sanitaire de la Côte d'Ivoire fortement dominée par les maladies infectieuses et parasitaires. En effet, le paludisme est actuellement la première cause de consultation dans les formations sanitaires de base et représente $57 \%$ des pathologies déclarées au cours des consultations générales dans les formations sanitaires (PNLP, 2005). II constitue la première cause de mortalité chez les enfants de moins de 5 ans dans les services de pédiatrie (OMS, 2004).

Certaines de ces plantes sont sollicitées pour traiter plusieurs pathologies. On peut citer Alchornea cordifolia (7 maladies), Ageratum conyzoides (5 maladies), Phyllanthus amarus, Vernonia colorata, Ocimum gratissimum, Jatropha curcas et Trema guineensis (4 maladies). Beaucoup d'autres par contre ne sont recherchées que pour traiter une seule maladie pendant la même période. C'est le cas de Rauvolfia vomitoria, Anchomanes difformis, Palisota hirsuta, Paullinia pinnata. 
Tableau 1: Liste des plantes recensées dans la ville d'Abidjan et les pathologies qui leur sont associées

\begin{tabular}{|c|c|c|}
\hline Familles & Espèces végétales & Pathologies \\
\hline Amarantaceae & Alternanthera repens (L.) Link. & $\begin{array}{l}\text { Hémorroïde } \\
\text { Diabète }\end{array}$ \\
\hline Anarcadiaceae & $\begin{array}{l}\text { Spondias mombin } \mathrm{L} \text {. } \\
\text { Anacardium occidentale } \mathrm{L} \text {. } \\
\text { Mangifera indica } \mathrm{L} \text {. }\end{array}$ & $\begin{array}{l}\text { Fontanelle } \\
\text { Hypertension artérielle } \\
\text { Paludisme }\end{array}$ \\
\hline Annonaceae & Annona muricata $\mathrm{L}$. & Diabète \\
\hline \multirow[t]{3}{*}{ Apocynaceae } & Alstonia boonei De Wild. & $\begin{array}{l}\text { Paludisme } \\
\text { Diabète }\end{array}$ \\
\hline & Catharanthus roseus (L.) G. Don & $\begin{array}{l}\text { Hypertension artérielle } \\
\text { Diabète }\end{array}$ \\
\hline & Rauvolfia vomotoria Afzel. & Paludisme \\
\hline Araceae & Anchomanes difformis (Blume) Engl. & Diabète \\
\hline Asclepiadaceae & Calotropis procera (Ait.) Ait. $f$ & $\begin{array}{l}\text { Dermatoses } \\
\text { Fontanelle }\end{array}$ \\
\hline \multirow[t]{5}{*}{ Asteraceae } & Ageratum conyzoides L. & $\begin{array}{l}\text { Constipation } \\
\text { Hypertension artérielle } \\
\text { Diabète } \\
\text { Otite } \\
\text { Paludisme }\end{array}$ \\
\hline & Bidens pilosa L. & $\begin{array}{l}\text { Diarrhée } \\
\text { Hémorroïde } \\
\text { Toux }\end{array}$ \\
\hline & Chromolaena odorata (L.) R. M. King & $\begin{array}{l}\text { Paludisme } \\
\text { Toux }\end{array}$ \\
\hline & Eclipta prostrata (L.) L. & $\begin{array}{l}\text { Fontanelle } \\
\text { Diabète }\end{array}$ \\
\hline & Vernonia colorata (Willd.) Drake & $\begin{array}{l}\text { Dermatoses } \\
\text { Hypertension artérielle } \\
\text { Paludisme } \\
\text { Diabète }\end{array}$ \\
\hline Bombacaceae & $\begin{array}{l}\text { Adansonia digitata L. } \\
\text { Ceiba pentandra (L.) Gaerth. }\end{array}$ & $\begin{array}{l}\text { Hypertension artérielle } \\
\text { Hypertension artérielle }\end{array}$ \\
\hline \multirow[t]{2}{*}{ Caesalpiniaceae } & $\begin{array}{l}\text { Afzelia Africana Sm. } \\
\text { Cassia alata L. }\end{array}$ & $\begin{array}{l}\text { Plaie de ventre } \\
\text { Constipation } \\
\text { Dermatoses } \\
\text { Paludisme } \\
\text { Hypertension artérielle }\end{array}$ \\
\hline & Cassia occidentalis L. & $\begin{array}{l}\text { Paludisme } \\
\text { Hypertension artérielle }\end{array}$ \\
\hline Caricaceae & Carica papaya L. & $\begin{array}{l}\text { Fièvre typhoïde } \\
\text { Parasitose }\end{array}$ \\
\hline Combretaceae & Anogeissus leiocarpus (DC) Guill. \& Perr. & Diabète \\
\hline Commelinaceae & Palisota hirsuta K. (Thunb.) Schum. ex Engl. & Rhumatisme \\
\hline Crassulaceae & Kalanchoe crenata (Andr.) Haw. & $\begin{array}{l}\text { Hémorroïde } \\
\text { Sinusite } \\
\text { Toux }\end{array}$ \\
\hline Cucurbitaceae & Momordica charantia L. & $\begin{array}{l}\text { Paludisme } \\
\text { Diabète }\end{array}$ \\
\hline \multirow[t]{5}{*}{ Euphorbiaceae } & Alchornea cordifolia (Schum. \& Thonn.) Müll. Arg. & $\begin{array}{l}\text { Anémie } \\
\text { Diabète } \\
\text { Hypertension artérielle } \\
\text { Paludisme } \\
\text { Perte blanche } \\
\text { Plaie de ventre } \\
\text { Toux }\end{array}$ \\
\hline & Euphorbia hirta L. & $\begin{array}{l}\text { Diarrhée } \\
\text { Diabète }\end{array}$ \\
\hline & Jatropha curcas L. & $\begin{array}{l}\text { Hémorroïde } \\
\text { Paludisme } \\
\text { Plaie de ventre } \\
\text { Diabète }\end{array}$ \\
\hline & Phyllanthus amarus Schum. \& Thonn. & $\begin{array}{l}\text { Diabète } \\
\text { Fièvre typhoïde } \\
\text { Hypertension artérielle } \\
\text { Paludisme }\end{array}$ \\
\hline & $\begin{array}{l}\text { Phyllanthus urinaria } \mathrm{L} \text {. } \\
\text { Ricinus communis } \mathrm{L} \text {. }\end{array}$ & $\begin{array}{l}\text { Hypertension artérielle } \\
\text { Hypertension artérielle }\end{array}$ \\
\hline Lamiaceae & Ocimum gratissimum $\mathrm{L}$. & $\begin{array}{l}\text { Dermatoses } \\
\text { Sinusite } \\
\text { Diabète } \\
\text { Hypertension artérielle }\end{array}$ \\
\hline Lauraceae & $\begin{array}{l}\text { Solenostemon monostachyus (P. Beauv.) Briq. } \\
\text { Persea americana Mill. }\end{array}$ & $\begin{array}{l}\text { Hémorroïde } \\
\text { Hypertension artérielle }\end{array}$ \\
\hline Liliaceae & Allium sativum $\mathrm{L}$. & Hypertension artérielle \\
\hline
\end{tabular}


Suite tableau 1

\begin{tabular}{|c|c|c|}
\hline $\begin{array}{l}\text { Loganiaceae } \\
\text { Malvaceae }\end{array}$ & $\begin{array}{l}\text { Anthocleista djalonensis A. Chev. } \\
\text { Hibiscus sabdariffa L. } \\
\text { Gossypium barbadense L. }\end{array}$ & $\begin{array}{l}\text { Diabète } \\
\text { Hypertension artérielle } \\
\text { Constipation } \\
\text { Otite }\end{array}$ \\
\hline Meliaceae & Azadirachta indica A. Juss. & $\begin{array}{l}\text { Paludisme } \\
\text { Hypertension artérielle }\end{array}$ \\
\hline Mimosaceae & $\begin{array}{l}\text { Albizia adianthifolia (Schum.) Whight } \\
\text { Parkia biglobosa (Jacq.) Benth. }\end{array}$ & $\begin{array}{l}\text { Hypertension artérielle } \\
\text { Hypertension artérielle } \\
\text { Diabète }\end{array}$ \\
\hline Moraceae & Ficus exasperata M. Vahl. & Dermatoses \\
\hline Myrtaceae & Psydium guajava L. & $\begin{array}{l}\text { Diarrhée } \\
\text { Hypertension artérielle }\end{array}$ \\
\hline Nyctaginaceae & Boerhavia diffusa L. & Angine \\
\hline Periplocaceae & Parquetina nigrescens (Afz.) Bul. & Diarrhée \\
\hline Poaceae & Bambusa vulgaris Schrad. ex Wendl. & $\begin{array}{l}\text { Fièvre typhoïde } \\
\text { Hypertension artérielle } \\
\text { Paludisme }\end{array}$ \\
\hline & $\begin{array}{l}\text { Cymbopogon citratus (DC.) Stapf } \\
\text { Imperata cylindrica L. } \\
\text { Saccharum officinarum L. }\end{array}$ & $\begin{array}{l}\text { Paludisme } \\
\text { Hypertension artérielle } \\
\text { Hypertension artérielle }\end{array}$ \\
\hline Rubiaceae & $\begin{array}{l}\text { Mitracarpus scaber Zucc. } \\
\text { Nauclea latifolia Sm. } \\
\text { Morinda lucida Benth. }\end{array}$ & $\begin{array}{l}\text { Dermatoses } \\
\text { Diabète } \\
\text { Hypertension artérielle }\end{array}$ \\
\hline Rutaceae & Fagara macrophylla Engl. & Hypertension artérielle \\
\hline Sapindaceae & Paullinia pinnata $\mathrm{L}$. & Rhumatisme \\
\hline Solanaceae & Solanum americanum Mill.. & $\begin{array}{l}\text { Anémie } \\
\text { Sinusite }\end{array}$ \\
\hline Steculiaceae & Cola nitida (Vent.) Schott \& Endl. & Hypertension \\
\hline Ulmaceae & Trema guineensis (Schum. \& Thonn.) Ficalho & $\begin{array}{l}\text { Anémie } \\
\text { Hypertension artérielle } \\
\text { Paludisme }\end{array}$ \\
\hline Verbenaceae & Tectona grandis L. f. & $\begin{array}{l}\text { Paludisme } \\
\text { Diabète } \\
\text { Fièvre typhoïde }\end{array}$ \\
\hline
\end{tabular}

L'hypertension artérielle et le diabète sont deux maladies relativement nouvelles pour les populations ivoiriennes comme pour celles des autres pays en développement de l'Afrique et du monde. Elles ont donc été difficilement nommées parce qu'elles restent encore mal connues de la pharmacopée ivoirienne et parce que la médecine traditionnelle ne dispose pas de moyens permettant de les diagnostiquer. Si pour l'hypertension artérielle, les acteurs de la médecine traditionnelle s'en remettent aux résultats des analyses de la médecine moderne et n'y ont actuellement pas encore trouvé de nom, pour le diabète surtout la forme sucrée par contre, il existe quelques indications pour diagnostiquer la maladie. Pour les populations Baoulé au Centre de la Côte d'Ivoire, le diabétique fait des urines incessantes au cours de la journée. Chez les Agni à l'Est et les Malinké au Nord, l'urine du diabétique attire les fourmis en raison de la présence de sucres dans celle-ci. C'est pourquoi le peuple Malinké nomme cette maladie “Soukarobannan " c'est-à-dire maladie du sucre. Pour les Dida au Sud, le diabétique fait des urines abondantes et moussantes, de coloration jaune foncée. En plus, les tradithérapeutes Dida identifient deux formes de diabète. La forme mâle caractérisée par un amaigrissement du patient et la forme femelle dont les patients sont obèses.

Comme on le constate, de ces deux pathologies, le diabète est celle qui est mieux connue par la médecine traditionnelle en Côte d'Ivoire. Cependant, pour proposer un traitement à tout patient présentant l'une ou l'autre des maladies, tous les tradithérapeutes exigent le diagnostic d'un médecin. Il s'agit là d'une parfaite collaboration entre deux médecines qui sont appelées à être complémentaires.

Au cours de cette étude, 39 plantes ont été inventoriées pour traiter l'hypertension artérielle et le diabète, soit $67,25 \%$ de l'ensemble des 
plantes recensées (Tableau 2). Ces deux maladies représentent $10,50 \%$ des pathologies pour lesquelles les populations ont recours aux plantes médicinales. II s'agit là d'un échantillonnage qui pourrait traduire la prévalence réelle de ces maladies au sein des populations démunies de la ville d'Abidjan et, partant, de celles de la Côte d'Ivoire. Mais elle reste certainement sous estimée parce qu'elle ne reflète pas le nombre réel d'hypertendus et de diabétiques dans la ville, la plupart des personnes ignorant leur statut. Plus de la moitié des plantes médicinales inventoriées sont recherchées contre ces deux maladies, ce qui traduit à la fois la diversité des plantes antihypertensives et antidiabétiques proposées aux patients et l'importance que ces patients accordent auxdites plantes.

Tableau 2: Espèces recherchées contre l'hypertension artérielle et le diabète et leurs proportions

\begin{tabular}{|c|c|c|c|c|}
\hline Espèces & Pathologies & $\begin{array}{c}\text { Proportion } \\
(\%)\end{array}$ & Organes & Préparation \\
\hline Adansonia digitata $\mathrm{L}$. & Hypertension artérielle & 18,07 & Feuille & Sauce \\
\hline \multirow[t]{2}{*}{ Ageratum conyzoides L. } & Hypertension artérielle & 12,04 & Feuille & Décocté \\
\hline & Diabète & 42,16 & Feuille & Décocté \\
\hline Albizia adianthifolia (Schum) Whight & Hypertension artérielle & 12,04 & Racine & Décocté \\
\hline \multirow[t]{2}{*}{ Alchornea cordifolia (Schum. \& Thonn.) Müll. Arg. } & Diabète & 36,14 & Feuille & Décocté \\
\hline & Hypertension artérielle & 18,07 & Feuille & Décocté \\
\hline Allium sativum $\mathrm{L}$. & Hypertension artérielle & 18,04 & Bulbe & Décocté \\
\hline \multirow[t]{2}{*}{ Alstonia boonei De Wild. } & Dibète & 24,09 & Feuille & Décocté \\
\hline & & 18,07 & Écorce & Macéré \\
\hline Alternanthera repens (L.) Link. & Diabète & 18,70 & Feuille & Décocté \\
\hline \multirow[t]{2}{*}{ Anacardium occidentale L. } & Hypertension artérielle & 30,12 & Écorce de tige & Macéré \\
\hline & & 12,04 & Écorce de tige & Décocté \\
\hline Anchomanes difformis (Blume) Engl. & Diabète & 18,07 & Racine & Macéré \\
\hline Annona muricata $\mathrm{L}$. & Diabète & 12,04 & Feuille & Décocté \\
\hline Anogeiossus leiocarpus (DC) Guill. \& Perr. & Diabète & 12,04 & Feuille & Décocté \\
\hline Anthocleista djalonensis A. Chev. & Diabète & 18,07 & Feuille & Décocté \\
\hline Azadirachta indica A. Juss. & Hypertension artérielle & 48,19 & Feuille & Décocté \\
\hline Bambusa vulgaris Schrad. ex Wendl. & Hypertension artérielle & 06,02 & Jeune pousse & Sauce \\
\hline Cassia alata L. & Hypertension artérielle & 12,04 & Feuille & Décocté \\
\hline Cassia occidentalis L. & Hypertension artérielle & 06,02 & Feuille & Décocté \\
\hline \multirow[t]{2}{*}{ Catharanthus roseus (L.) G. Don } & Hypertension artérielle & 42,16 & Racine & Décocté \\
\hline & Diabète & 36,14 & Plante entière & Décocté \\
\hline Ceiba pentandra (L.) Gaerth. & Hypertension artérielle & 12,04 & Feuille & Décocté \\
\hline Cola nidida (Vent.) Schott \& Endl. & Hypertension artérielle & 12,04 & Feuille & Décocté \\
\hline Eclipta prostrata L. & Diabète & 12,04 & Feuille & Décocté \\
\hline Euphorbia hirta L. & Diabète & 18,07 & Feuille & Décocté \\
\hline Fagara macrophylla Engl. & Hypertension artérielle & 18,07 & Écorce de tige & Décocté \\
\hline Hibiscus sabdariffa L. & Hypertension artérielle & 42,16 & Corolle & Décocté \\
\hline Imperata cylindrica L. & Hypertension artérielle & 06,02 & Racine & Macéré \\
\hline Jatropha curcas L. & Diabète & 06,02 & Graine & Décocté \\
\hline Momordica charantia L. & Diabète & 18,07 & Feuille, tige & Macéré \\
\hline Morinda lucida Benth. & Hypertension artérielle & 18,07 & Feuille & Décocté \\
\hline Nauclea latifolia Sm. & Diabète & 24,09 & Feuille, tige & Décocté \\
\hline \multirow[t]{2}{*}{ Ocimum gratissimum L. } & Hypertension artérielle & 18,07 & Feuille & Décocté \\
\hline & Diabète & 12,04 & Feuille & Décocté \\
\hline \multirow[t]{3}{*}{ Parkia biglobosa (Jacq.) Benth. } & Hypertension artérielle & 06,02 & Feuille & Décocté \\
\hline & & 24,09 & Graine & Sauce \\
\hline & Diabète & 18,07 & Feuille & Décocté \\
\hline Persea americana Mill. & Hypertension artérielle & 24,09 & Feuille & Décocté \\
\hline \multirow[t]{2}{*}{ Phyllanthus amarus Schum. \& Thonn. } & Diabète & 48,19 & Plante entière & Décocté \\
\hline & Hypertension artérielle & 42,16 & Feuille & Pâte \\
\hline Phyllanthus urinaria L. & Hypertension artérielle & 30,12 & Feuille & Décocté \\
\hline Psydium guajava L. & Hypertension artérielle & 12,04 & Feuille & Décocté \\
\hline Ricinus communis L. & Hypertension artérielle & 06,02 & Feuille & Décocté \\
\hline Saccharum officinarum $\mathrm{L}$. & Hypertension artérielle & 06,02 & Feuille & Décocté \\
\hline Tectona grandis $\mathrm{L}$. & Diabète & 18,07 & Feuille & Décocté \\
\hline Trema guineensis (Schum. \& Thonn.) Ficalho & Hypertension artérielle & 42,16 & Feuille & Décocté \\
\hline \multirow[t]{2}{*}{ Vernonia colorata (Willd.) Drake } & Hypertension artérielle & 12,04 & Feuille & Décocté \\
\hline & Diabète & 06,02 & Feuille & Décocté \\
\hline
\end{tabular}


Parmi les 39 plantes, 27 sont antihypertensives. Elles appartiennent à 25 genres et 18 familles. Les familles les plus représentées sont les Euphorbiaceae (4 espèces), les Poaceae (3 espèces) puis les Asteraceae, Bombacaceae, Caesalpiniaceae et Mimosaceae (2 espèces). Les plantes les plus recherchées sont Azadirachta indica, Catharanthus roseus, Phyllanthus amarus, Hibiscus sabdariffa, Parkia biglobosa, Trema guineensis, Anacardium occidentale et Phyllanthus urinaria (Tableau 2). Ainsi donc, bien que les acteurs de la médecine traditionnelle ne maîtrisent pas parfaitement I'hypertension artérielle aussi bien dans sa dénomination que dans son diagnostic, ils ont pu trouver dans leur environnement suffisamment de plantes pour faire face à ce mal.

Des enquêtes ethnomédicinales réalisées dans d'autres pays en voie de développement ont signalé l'utilisation de certaines des plantes contre ce mal. Les plus récentes indiquent Persea americana, Hibiscus sabdariffa et Cola nitida (Lans, 2006). Pour d'autres plantes, l'activité antihypertensive a, en plus, été établie par des études pharmacologiques. C'est le cas de Hibiscus sabdariffa (Ajay, 2007 ; Mojiminiyi, 2007).

Dix neuf (19) plantes sont antidiabétiques. Elles appartiennent à 19 genres et 13 familles, les familles les plus représentées étant les Euphorbiaceae (4 espèces), les Asteraceae (3 espèces) et les Apocynaceae (2 espèces). Les plus sollicitées sont Phyllanthus amarus, Ageratum conyzoides, Catharanthus roseus et Alchornea cordifolia (Tableau 2). Comme dans le cas de l'hypertension artérielle, certaines des plantes ont été signalées par des enquêtes ethnomédicinales récentes dans le traitement du diabète. Parmi ces plantes figurent Allium sativum (Eddouks et al., 2002), Catharanthus roseus et Momordica charantia (Lans, 2006). L'activité antidiabétique de certaines de ces plantes a également été prouvée expérimentalement. C'est le cas de Ceiba pentandra (Ladeji et al., 2003), Vernonia colorata (Sy et al., 2007), Parkia biglobosa (Odetola et al., 2006) et Phyllanthus amarus (Srividya \& Periwals, 1995). Ces résultats qui confirment l'activité biologique de ces plantes expliquent, en partie, leur utilisation dans le traitement de l'hypertension artérielle et du diabète par les populations de la ville d'Abidjan. Des études similaires sont nécessaires, dans un premier temps, pour les espèces dont les proportions observées au cours de la présente étude sont élevées afin de confirmer ou non leurs activités biologiques et étendre par la même occasion la gamme de plantes antihypertensives et antidiabétiques. Cela résoudrait partiellement le problème de santé en Côte d'lvoire, du moins pour ce qui est de l'hypertension artérielle et du diabète.

Six (6) plantes sont utilisées pour traiter aussi bien l'hypertension artérielle que le diabète (Tableau 3). II s'agit de Catharanthus roseus, Ageratum conyzoides, Vernonia colorata, Alchornea cordifolia, Phyllanthus amarus et Parkia biglobosa. Ce résultat montre bien le lien étroit entre l'hypertension artérielle et le diabète expliquant ainsi le fait que certaines plantes que nous avons notées contre l'hypertension artérielle (Annona muricata, Phyllanthus urinaria) soient également signalées ailleurs contre le diabète (Lans, 2006). Les six plantes que nous avons signalées contre ces pathologies émergentes ouvrent des perspectives intéressantes dans la recherche de médicaments pouvant être prescrits pour traiter simultanément l'hypertension artérielle et le diabète.

Tableau 3: Espèces utilisées pour traiter à la fois l'hypertension artérielle et le diabète

\begin{tabular}{llll}
\hline Espèces & \multicolumn{1}{c}{ Pathologies } & organes & Préparation \\
\hline Catharanthus roseus (L.) G. Don & Hypertension artérielle & Racine & Décocté \\
& Diabète & Plante entière & Décocté \\
Ageratum conyzoides L. & Hypertension artérielle & Feuille & Décocté \\
& Diabète & Feuille & Décocté \\
Vernonia colorata (Willd.) Drake & Hypertension artérielle & Feuille & Décocté \\
& Diabète & Feuille & Décocté \\
Alchornea cordifolia (Schum. \& Thonn.) Müll. Arg. & Diabète & Feuille & Décocté \\
& Hypertension artérielle & Feuille & Décocté \\
Phyllanthus amarus Schum. \& Thonn. & Diabète & Plante entière & Décocté \\
& Hypertension artérielle & Feuille & Pâte \\
Ocimum gratissimum L. & Hypertension artérielle & Feuille & Décocté \\
& Diabète & Feuille & Décocté \\
Parkia biglobosa (Jacq.) Benth. & Hypertension artérielle & Feuille & Décocté \\
& & Graine & Sauce \\
\hline
\end{tabular}


Ces résultats montrent que l'hypertension artérielle et le diabète qui sont des maladies émergentes liées au développement et au changement du mode de vie, sont présents aujourd'hui au sein des populations, particulièrement de celles de la ville d'Abidjan. Ils indiquent également que les patients hypertendus et les diabétiques ont recours à la médecine traditionnelle pour se traiter, ce qui justifie le nombre important et la gamme variée de plantes utilisées contre ces deux maladies et commercialisées sur les marchés de la ville d'Abidjan. L'intérêt que portent les patients pour les plantes tient du fait que la pharmacopée, pour ce qui est du traitement de ces deux affections, revient nettement moins chère. De plus, le traitement est moins contraignant par rapport à celui de la médecine moderne. À l'analyse des résultats, on s'aperçoit que ce sont les malades hypertendus qui ont plus recours aux plantes médicinales. Cette affection, à cause de son caractère violent et de la mort qu'elle peut donner à tout instant à travers les atteintes cardiovasculaires, est de plus en plus vite diagnostiquée. En effet, selon Koffi et al. (2001), en Côte d'Ivoire, $15 \%$ des motifs de consultation dans certaines structures sanitaires de premier contact sont dus à l'hypertension artérielle. Ce n'est pas le cas du diabète qui est plutôt considéré comme une maladie silencieuse. Les consultations contre l'hypertension artérielle sont donc plus nombreuses que celles contre le diabète de sorte que pour l'hypertension artérielle, on savait en 2000 que $13,9 \%$ de la population ivoirienne et $21 \%$ de la population de la ville d'Abidjan étaient concernées par cette maladie (Koffi, 2007). Par contre, pour le diabète, les seules données nationales de prévalence disponibles datent de 1979 et indiquaient déjà un niveau élevé à $5,7 \%$ de la morbidité diabétique (Oga et al., 2006). En considérant ces données, on pourrait affirmer que les résultats que nous avons obtenus reflètent quelque peu la prévalence actuelle de ces deux maladies en Côte d'Ivoire, l'hypertension étant plus répandue que le diabète. D'ailleurs, les travaux de Koffi (2007) indiquent que la famille des antihypertenseurs a représenté en 2005 près de $15 \%$ du chiffre d'affaire global des médicaments dispensés à l'Institut de Cardiologie d'Abidjan (Côte d'Ivoire). Mais le diagnostic n'étant pas fait pour toute la population ivoirienne, il reste difficile d'affirmer que l'une des maladies prédomine l'autre.

Pour cette étude, les feuilles sont les organes les plus utilisés dans les préparations médicamenteuses. Ce sont les organes disponibles tout au long de l'année, d'accès et de prélèvement faciles et qui sont de manipulation aisée. Elles sont suivies par les écorces de tige puis les racines et les pièces florales. Tous ces organes sont préparés principalement sous la forme de décocté. C'est le mode de préparation le plus courant dans les traitements par les plantes pour la grande majorité des tradithérapeutes. La macération et la trituration sont également indiquées. Ces préparations sont pratiquement toutes prescrites en boisson. Cette prescription peut s'expliquer par le fait que les deux maladies sont liées à des organes profonds. Pour les atteindre, tout composé doit transiter par l'appareil digestif pour en faciliter son assimilation.

\section{Conclusion}

Les investigations que nous avons menées ont permis de comprendre que l'hypertension artérielle et le diabète, deux maladies encore inconnues jusqu'au milieu du $20^{{ }^{e ̀ m e}}$ siècle en Côte d'Ivoire, touchent de plus en plus de populations dans ce pays. Bien que ne disposant pas de moyens performants leur permettant de diagnostiquer ces maladies, les guérisseurs traditionnels trouvent dans les plantes les moyens de les traiter. Le nombre de plantes recensées au cours de cette étude en est une parfaite illustration. Le diabète qui est la maladie la mieux connue par la pharmacopée ivoirienne est celle pour laquelle les plantes inventoriées ne sont pas nombreuses. De toute évidence, la situation de ces deux maladies reste encore sous estimée. II convient dès à présent de procéder au dépistage systématique de ces maladies au sein de la population nationale afin de permettre à la médecine moderne et à la pharmacopée traditionnelle de lutter ensemble contre ces fléaux.

\section{Remerciements}

Nous tenons à remercier, ici, toutes les femmes commerçantes de plantes médicinales des marchés de Yopougon, Abobo et Port Bouët pour leur franche collaboration à cette étude. Nous remercions également les patients acheteurs et utilisateurs de plantes médicinales qui ont bien voulu se prêter à nos questions. Nous remercions, enfin, le Professeur Aké-Assi Laurent et le Centre National de Floristique de l'Université de CocodyAbidjan pour leur contribution à l'identification des plantes inventoriées. 


\section{Références citées}

Ajay M., Chai H.J., Mustafa A.M., Gilani A.H. \& Mustafa M.R., 2007. Mechanisms of antihypertensive effect of Hibiscus sabdariffa L. calyces. J. Ethnopharmacol. 109: 388-393.

Aké Assi L., 1984. Flore de la Côte-d'Ivoire: étude descriptive et biogéographique, avec quelques notes ethnobotaniques. Tomes I, II, III. Thèse Doctorat ès-Sciences Naturelles, FAST. Université d'Abidjan (Côte-d'Ivoire); 1205 pp.

Anonyme, 2003. Hypertension artérielle et ses complications. Abidjan (Côte d'Ivoire): Institut de Cardiologie d'Abidjan; 183 pp.

Comoé K.C., Sess D. \& Guelain J., 1993. Etude de l'hypertension artérielle en milieu urbain de Côte d'Ivoire. Med. Trop. 53 (2): 173-179.

Diehl M.S., Kamanzi Atindehou K., Téré H. \& Betschart B., 2004. Prospect for anthelminthic plants in the Ivory Coast using ethnobotanical criteria. J. Ethnopharmacol. 95: 277-284.

Eddouks M., Maghrani M., Lemhadri A., Ouahid M.-L. \& Jouad H., 2002. Ethnophramacological survey of medicinal plants used for the traitment of diabetes mellitus, hypetension and cardiac diseases in the South-East region of Morocco (Tafilalet). J. Ethnopharmacol. 82: 97-103.

Fennell C.W., Light M.E., Sparg S.G., Stafford G.I. \& van Staden J., 2004. Assessing African medicinal plants for efficacy and safety: agricultural and storage practices. $J$. Ethnopharmacol. 95: 113-121.

Hamill F.A., Apio S., Mubiru N.K., Bukenya-Ziraba R., Mosango M., Maganyi O.W. \& Soejarto D.D., 2003. Traditional herbal drugs of Southern Uganda, II: literature analysis and antimicrobial assays. J. Ethnopharmacol. 84: 57-78.

Hutchinson J. \& Dalziel J.M., 1954. Flora of West Tropical Africa. Crown Agents for Oversea Government and Administratons Millbank, London (England); 828 pp.

Hutchinson J. \& Dalziel J.M., 1963. Flora of West Tropical Africa. Crown agents for Oversea Government and Administratons Millbank, London (England); 544 pp.

Kamanzi Atindehou K., Koné M., Terreaux C., Traoré D., Hostettmann K. \& Dosso M., 2002.
Evaluation of the Antimicrobial Potential of Medicinal Plants from the Ivory Coast. Phytother. Res. 16 (5): 497-502.

Kamatenesi-Mugisha M. \& Oryem-Origa H., 2007. Medicinal plants used to induce labour during childbirth in western Uganda. J. Ethnopharmacol. 109: 1-9.

Koffi A., 2007. Prise en charge des patients adultes atteints d'hypertension artérielle à l'Institut de Cardiologie d'Abidjan. Abidjan (Côte d'Ivoire): Rapport ICA; 6 pp.

Koffi N.M., Sally S.J., Kouamé P., Silué K. \& Diarra Nama A.J., 2001. Faciès de l'hypertension artérielle en milieu professionnel à Abidjan. Med. Afr. 6: 48.

Kouamé N.F., 1998. Influence de l'exploitation forestière sur la végétation et la flore de la forêt classée du Haut-Sassandra (Centre-Ouest de la Côte d'Ivoire). Thèse 3ème cycle. Université de Cocody-Abidjan (Côte d'Ivoire); 227 pp.

Ladeji O., Omekara I. \& Solomon M., 2003. Hypoglycemic properties of aqueous bark extract of Ceiba pentandra in streptozotocin-induced diabetic rats. J. Ethnopharmacol. 84: 139-142.

Lans C.A., 2006. Ethnomedicines used in Trinidad and Tobago for urinary problems and diabetes mellitus. J. Ethnobiol. Ethnomed. 2: 45.

Lebrun J.-P. \& Stork A.L., 1991. Enumération des plantes à fleurs d'Afrique Tropicale : Annonaceae à Pandaceae Gamopétales: Ericaceae à Lamiaceae. CJB, Génève (Suisse); 249 pp.

Lebrun J.-P. \& Stork A.L., 1992. Enumération des plantes à fleurs d'Afrique Tropicale: Chrysobalanaceae à Apiaceae. CJB, Génève (Suisse); 257 pp.

Lebrun J.-P. \& Stork A.L., 1995. Enumération des plantes à fleurs d'Afrique Tropicale. Monocotylédones : Limnocharitaceae à Poaceae. CJB, Génève (Suisse); 314 pp.

Lebrun J.-P. \& Stork A.L., 1997. Enumération des plantes à fleurs d'Afrique Tropicale. Gamopétales: Ericaceae à Lamiaceae. CJB, Génève (Suisse); 712 pp.

Mojiminiyi F.B.O., Dikko M., Muhammad B.Y., Ojobor P.D., Ajagbonna O.P., Okolo R.U., Igokwe U.V., Mojiminiyi U.E., Fagbemi M.A., Bello S.O. \& Anga T.J., 2007. Antihypertensive effect of an 
aqueous extract of the calyx of Hibiscus sabdariffa. Fitoterapia 78: 292-297.

Odetola A.A., Akinloye O., Egunjobi C., Adekunle W.A. \& Ayoola A.O., 2006. Possible antidiabetic and antihyperlipidaemic effect of fermented Parkia biglobosa (Jacq.) extract in alloxaninduced diabetic rats. Clin. Exp. Pharmacol. Physiol. 33: 808-812.

Oga A.S.S., Tebi A., Aka J., Adouéni K.V., Malan K.A., Kouadio L.P. \& Lokrou A., 2006. Le diabète sucré diagnostiqué en Côte d'Ivoire: des particularités épidémiologiques. Med. Trop. 66: 241-246.

OMS, 2004. Stratégie OMS de coopération avec la République de Côte d'Ivoire 2004-2007. OMS,
Bureau Régional pour l'Afrique, Brazzaville (République du Congo); 39 pp.

PNLP, 2005. Document de politique nationale pour la lutte contre le paludisme. Abidjan (Côte d'Ivoire): Programme National de Lutte contre le Paludisme; 18 pp.

Srividya N. \& Periwals S., 1995. Diuretic, hypotensive and hypoglycaemic effect of Phyllanthus amarus. Indian J. Exp. Biol. 33 (11): 861-864.

Sy G.Y., Cissé A., Nongonierma R.B., Sarr M., Mbodj N.A. \& Faye B., 2007. Hypoglycaemic and antidiabetic activity of acetonic extract of Vernonia colorata leaves in normoglycaemic and alloxaninduced rats. J. Ethnopharmacol. 98: 171-175. 